\title{
Patient selection for trans-catheter mitral valve repair vs. replacement: ongoing indications and glimpse to the future
}

\author{
Andrea Scotti', Michele Galasso², Alberto Margonato ${ }^{2}$, Cosmo Godino ${ }^{2}$ \\ 'Department of Cardiac Thoracic Vascular Sciences and Public Health, University of Padua Medical School, Padua 35128, Italy. \\ ${ }^{2}$ Cardio-Thoracic-Vascular Department, San Raffaele Scientific Institute, Milan 20132, Italy.
}

Correspondence to: Dr. Cosmo Godino, Cardio-Thoracic-Vascular Department, San Raffaele Scientific Institute. Via Olgettina 60, Milan 20132, Italy. E-mail: godino.cosmo@hsr.it

How to cite this article: Scotti A, Galasso M, Margonato A, Godino C. Patient selection for trans-catheter mitral valve Repair versus Replacement: ongoing indications and glimpse to the future. Vessel Plus 2020;5:6.

http://dx.doi.org/10.20517/2574-1209.2020.68

Received: 7 Nov 2020 First Decision: 3 Dec 2020 Revised: 3 Dec 2020 Accepted: 9 Dec 2020 Published: 21 Jan 2021

Academic Editor: Manel Sabate Copy Editor: Monica Wang Production Editor: Jing Yu

\begin{abstract}
Transcatheter therapies for patients with severe mitral regurgitation are increasingly performed in recent years. While transcatheter mitral valve repair (TMVR) is now a consolidated intervention with $>100,000$ procedures performed worldwide, TMV replacement (TMVRpl) is in its early stages and can only be offered by a few centers. The complexity of mitral valve anatomy requires careful evaluation when selecting the best approach and the most suitable device. At present, the clinical experience with TMVRpl is still in a preliminary phase. Most of the patients treated with this approach thus far are high-risk candidates who have undergone a very restrictive clinical and anatomical selection. Therefore, it is difficult to correctly define which patients might benefit more from TMVRpl than from TMVR. We review the clinical, pathophysiological, and technical factors to suggest when to prefer TMVR instead of a replacement technique.
\end{abstract}

Keywords: Mitral regurgitation, transcatheter mitral valve repair, transcatheter mitral valve replacement

\section{INTRODUCTION}

Percutaneous procedures can be considered a safe alternative to surgical approaches in most patients affected by severe mitral regurgitation (MR). Although transcatheter mitral valve repair (TMVR) is

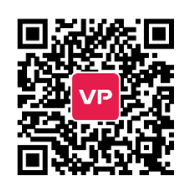




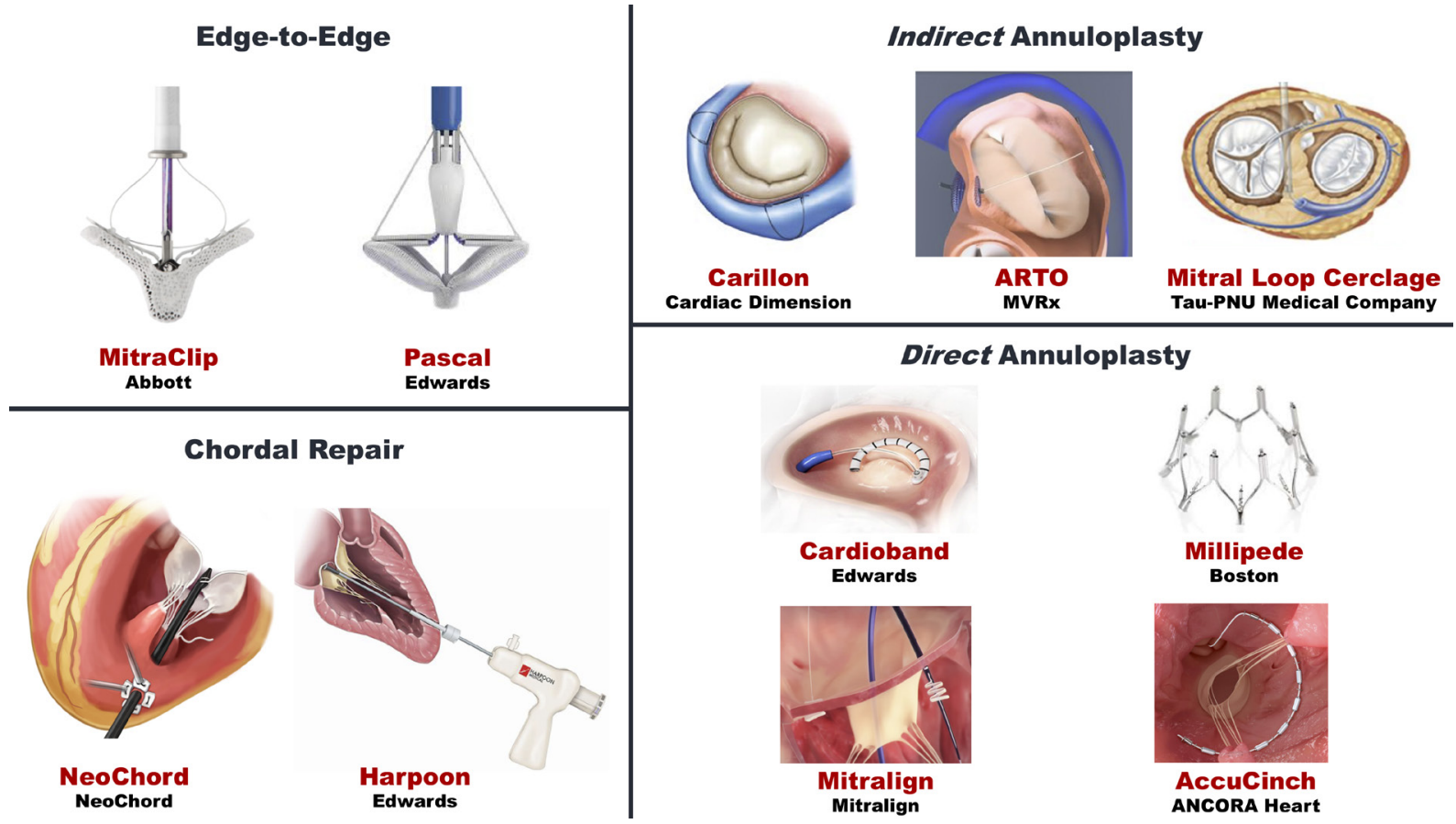

Figure 1. Transcatheter mitral valve repair devices

currently the first choice in most clinical settings, transcatheter mitral valve replacement (TMVRpl) will increasingly be a complementary technique in the near future. Several devices have been approved or are under investigation, permitting a customized strategy based on clinical and anatomical factors.

\section{TRANSCATHETER MITRAL VALVE REPAIR}

Taking inspiration from the surgical counterpart, we can classify percutaneous repair techniques [Figure 1] as follows:

-Edge-to-edge (Alfieri-stich) repair: MitraClip and PASCAL

-Percutaneous annuloplasty: Carillon, Cardioband, Millipede, Mitralign, ARTO, AccuCinch, and Mitral Loop Cerclage

-Chordal repair: NeoChord and HarpoonCords

The MitraClip (Abbott Laboratories, Menlo Park, California, USA) is a cobalt-chromium clip with two arms used to grasp and suture together the anterior and posterior valvular leaflets. It is the device with the widest clinical use ( $>100,000$ procedures worldwide) and proved to be safe and effective in both primary MR (PMR) and secondary MR (SMR) ${ }^{[1-3]}$. The PASCAL (Edwards Lifesciences, Irvine, CA) TMVR system is newer and differs from the previous one by an easy steerability, a larger implant size, and broader paddles that can be controlled individually ${ }^{[4]}$.

Percutaneous annuloplasty can be performed through the coronary sinus (indirect) or reaching the mitral valve from transseptal or retrograde LV access (direct). The indirect approach (Carillon, ARTO, Mitral Loop Cerclage Catheter System) feasibility is limited by unfavorable anatomy of the coronary sinus and its branch veins in a significant proportion of patients. Direct annuloplasty (Cardioband, Millipede, Mitralign, AccuCinch) involves the implantation of anchors around the mitral annulus tethered by the delivery system. 


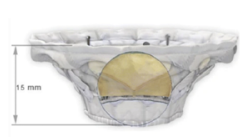

CardioValve Valtech (Edwards)

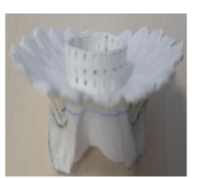

MValve Mvalve Tech

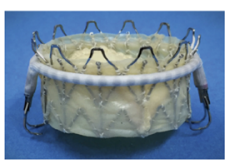

SATURN InnovHeart

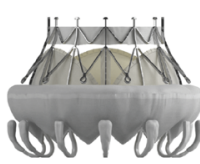

CardiAQ Edwards

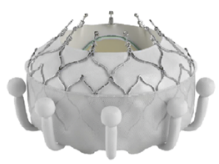

EVOQUE

Edwards

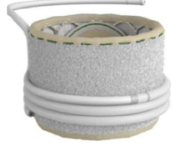

Sapien M3

Edwards

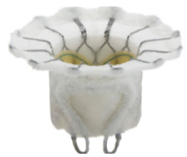

Fortis

Edwards

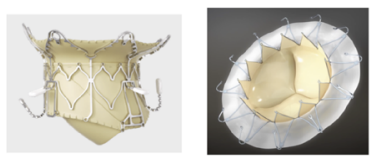

EPYGON
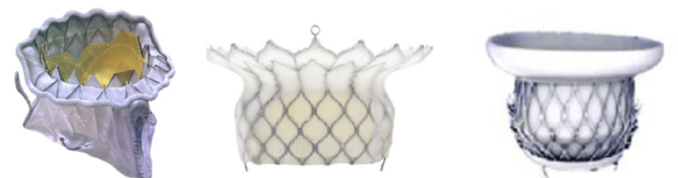

Tiara Neovasc

HighLife HighLife Medical

Mi-thos

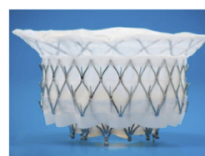

Intrepid

Medtronic

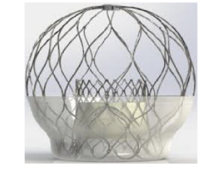

AltaValve 4C Medical nghai NewMed

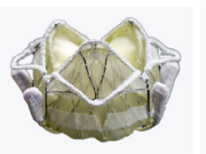

Caisson Livanoval

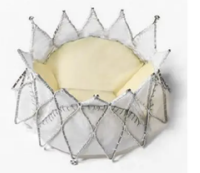

Corona Valcare
SINOMED

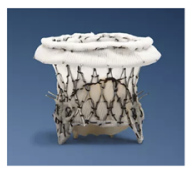

Permavalve Micro Interventional

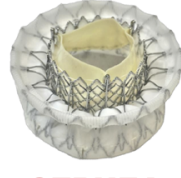

CEPHEA

Abbott

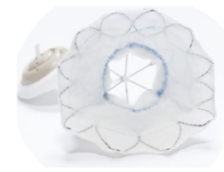

Tendyne Abbott

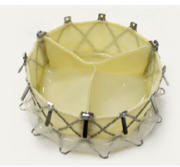

NaviGate NCSI

Others: - Braile - Direct Flow - MitrAssist - MitralHeal - ValveXchange - Transcat. Tech. - Lutter - Mehr - Mitralix - MitralTech - Mitracath - Mitralix - Nakostech - St George ATLAS - Venus - Verso - Transmural Systems

Figure 2. Transcatheter mitral valve replacement devices

Chordal repair (NeoChord, HarpoonCords) is obtained through a surgical transapical off-pump approach; no transarterial or transvenous systems are currently available. It is most commonly adopted in high-risk patients with PMR.

\section{TRANSCATHETER MITRAL VALVE REPLACEMENT}

TMVRpl is in its early stages and can only be offered by a few centers. This technology has the potential of obtaining predictable and reproducible results, and it is expected to grow rapidly as more clinical data become available.

A transcatheter aortic or pulmonary prosthesis, SAPIEN-XT (Edwards Lifesciences, Irvine, CA) or Melody valve (Medtronic, Minneapolis, MN), can be implanted in patients with severe MR and previous mitral surgery (bioprosthesis or annuloplasty) with valve-in-valve or valve-in-ring procedures or severe mitral annular calcification (valve-in-MAC) in selected individuals who are at too high risk for surgery.

In the case of native mitral valve, the worldwide experience regarding TMVRpl is still limited to slightly more than 500 procedures. All the available devices consist of a self-expanding frame that reproduces a trileaflet biological valve with xenogenic leaflets [Figure 2]. At the moment, the transapical access through anterolateral minithoracotomy is the most used approach: it establishes a great coaxiality with the mitral ring and permits the use of introducers large enough to deliver a much larger valve than the aortic ones. Some valves are built with a characteristic D-shape to conform to the physiological mitral anatomy. The peculiar conformation of the native valve undermines a successful deployment; the radial force is not uniform (as opposed to the aortic devices) and the risks of paravalvular leakage and prosthesis migration are real. For this purpose, different anchoring systems have been envisioned to hold the valve in place by attaching the anchors to the annulus, the subvalvular apparatus, or the left ventricular apex. 


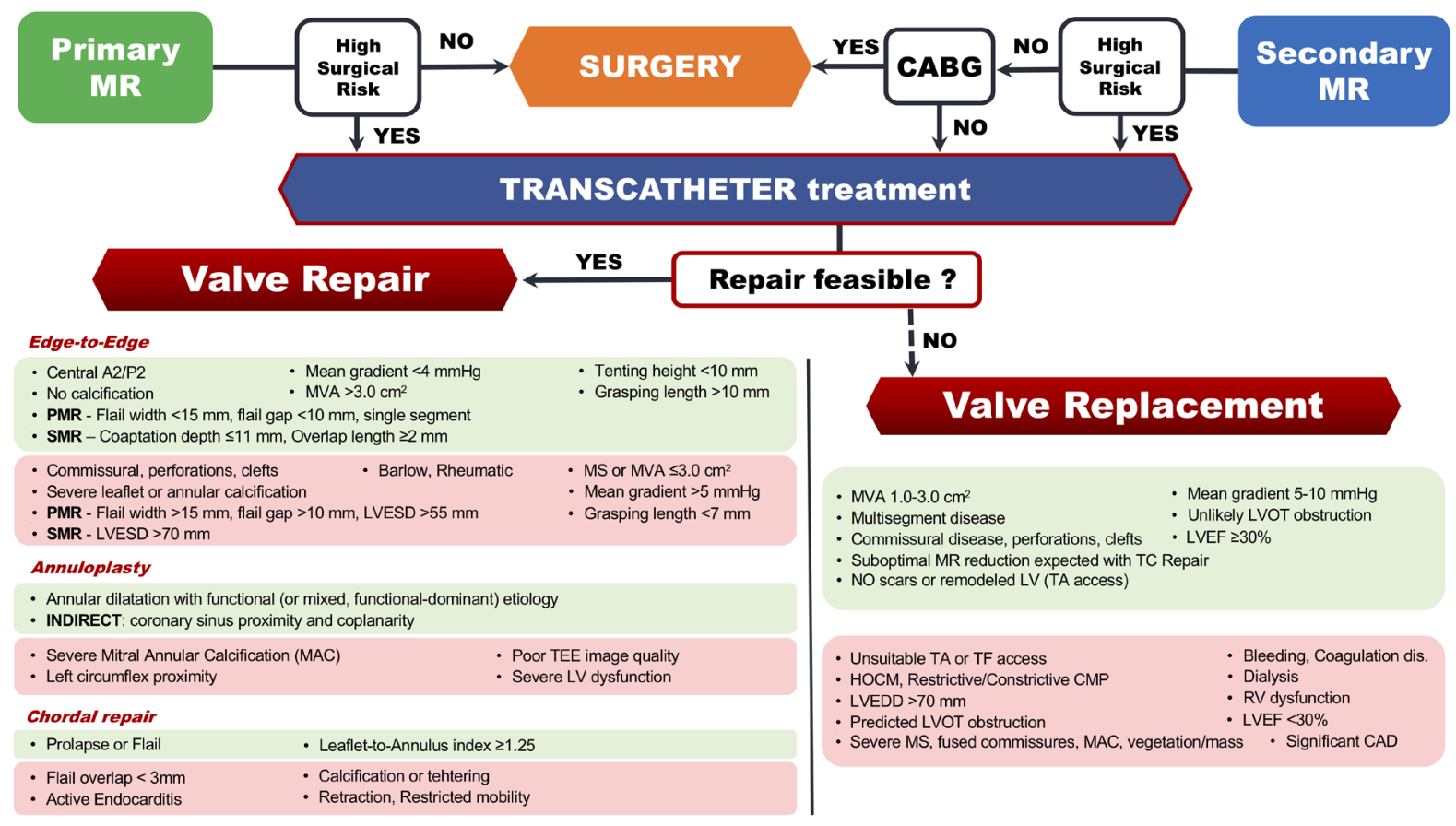

Figure 3. Interventional algorithm for mitral regurgitation. Light green box: favorable feature; light red box: less favorable features or unfeasibility; CABG: coronary artery bypass grafting; CAD: coronary artery disease; CMP: cardiomyopathy; HOCM: hypertrophic obstructive cardiomyopathy; LV: left ventricle; LVEDD: left ventricular end-diastolic diameter; LVEF: left ventricular ejection fraction; LVESD: left ventricular end-systolic diameter; LVOT: left ventricular outflow tract; MS: mitral stenosis; MVA: mitral valve area; PMR: primary mitral regurgitation; RV: right ventricle; SMR: secondary mitral regurgitation; TA: transapical; TC: transcatheter; TEE: transesophageal echocardiography; TF: transfemoral

\section{CLINICAL FACTORS}

\section{Primary mitral regurgitation (PMR)}

Surgical mitral valve repair, also through minimally invasive access, is effective, with excellent results at long-term follow-up, and represents the gold standard in the case of PMR [Figure 3]. Transcatheter therapies should be reserved for patients at high surgical risk, with reasonable life expectancy, and a considerable symptomatic burden (New York Heart Association class III or IV). These recommendations were driven mainly by analysis of the EVEREST (Endovascular Valve Edge-to-Edge REpair Study) trials and outcomes reported in the REALISM (Real World Expanded Multicenter Study of the MitraClip System) Registry (technical success $95 \%$, stroke $2.4 \%$, unplanned mitral valve surgery $0.8 \%$, and 30 -day mortality $6.3 \%)^{[5,6]}$. Edge-to-edge repair and chordal replacement can be offered to the patients who satisfy those criteria. In this setting, TMVRpl may actually be considered in the absence of favorable anatomy for TMVR techniques.

\section{Secondary mitral regurgitation (SMR)}

SMR is by definition associated with a certain degree of atrial or left ventricle (LV) abnormality. Annulus dilation is the main mechanism of atrial SMR and is induced by elevated atrial pressures and eventual atrial fibrillation ${ }^{[7]}$. A ventricular-secondary mechanism can be found when the LV is dilated and remodeled to the extent that causes mitral annulus widening and papillary muscles' displacement, which in turn tether the valve leaflets and avoid a competent coaptation. Whether MR is the "primum movens" or an epiphenomenon of LV disease may be assumed by estimating the contribution of LV function to the severity of MR. The ratio between effective regurgitant orifice area and LV end diastolic volume has been proposed as a discriminatory tool to identify proportionate ("true secondary") and disproportionate ("primum movens") $\mathrm{MR}^{[8]}$. 
High morbidity/mortality and suboptimal results undermine the surgical treatment (both repair and replacement) of SMR. The surgical approach is clearly indicated in the limited setting of patients with LV ejection fraction $(\mathrm{LVEF})>30 \%$ and needing concomitant coronary revascularization through coronary artery bypass grafting (class of recommendation $=$ I for LVEF $>30 \%$, IIa for LVEF $\leq 30 \%$ ) [Figure 3] ${ }^{[9]}$. Therefore, transcatheter therapies are widely used for SMR (technical success 97\% and 95.8\%, stroke 0.7\% and $1.4 \%$, unplanned mitral valve surgery $1 \%$ and $0 \%$, and 30 -day mortality $2.3 \%$ and $3.3 \%$, in COAPT and MITRA-FR trials, respectively ${ }^{[10,11]}$ ).

Edge-to-edge clips, annuloplasty devices, and TMVRpl valves can be used to treat patients with SMR after careful multi-disciplinary assessment ${ }^{[12]}$. The contrasting yet complementary results of the two available randomized trials on MitraClip implantation (COAPT and MITRA-FR) ${ }^{[10,11]}$ make it clear that careful evaluation of clinical and anatomical factors are of primary importance. The response to transcatheter techniques is expected to be less beneficial in the case of severe LV dilatation, long-standing pulmonary hypertension, concomitant atrial fibrillation, and severe chronic kidney disease ${ }^{[13]}$.

Optimal timing is crucial to obtain the best results in terms of survival and symptomatic benefit. However, a recent international registry has proved that TMVR may extend its therapeutic window even to patients affected by advanced heart failure ${ }^{[14]}$. This end-stage population can benefit the most from hemodynamic stabilization, symptomatic relief, and recovery from pulmonary hypertension. These factors can lead these patients to receiving elective heart transplantation in a good clinical status or even being delisted, no longer needing this advanced therapy.

\section{ANATOMICAL AND TECHNICAL FEATURES}

\section{Transcatheter mitral valve repair}

Criteria defining the optimal candidates for TMVR were initially derived from the EVEREST studies ${ }^{[15]}$, and subsequently validated and expanded from trials and large real-world registries [Figure 3]. From an anatomical point of view, a flail width $\leq 15 \mathrm{~mm}$, a flail gap $<10 \mathrm{~mm}$, and a single diseased segment are ideal for successful MitraClip placement in PMR. On the contrary, severely and diffusely thickened (5 mm in diastole) and retracted (rheumatic disease) or redundant leaflets (Barlow's type valve) contraindicate TMVR for PMR. The most important factors to be evaluated for SMR treatment are a sufficient leaflet tissue (grasping length $>10 \mathrm{~mm}$ ), a coaptation length $\geq 2 \mathrm{~mm}$, and a coaptation depth $\leq 11 \mathrm{~mm}$. Leaflets containing perforations, calcifications on the grasping zone, or deep cleft-like indentations are less likely to return good results. Multivariate logistic regression identified preprocedural mitral valve area $\leq 3 \mathrm{~cm}^{2}$ and mean mitral valve gradient $>4 \mathrm{mmHg}$ as independent predictors of overall procedural failure ${ }^{[16]}$.

Severe annular dilation should guide toward a direct or indirect annuloplasty device as first choice or complementary tool. In this case, an angio-CT scan should evaluate the proper size of the mitral valve annulus, the presence of calcifications, and the relations with the circumflex artery.

\section{Transcatheter mitral valve replacement}

The complexity of mitral anatomy with its D-shape and well-represented subvalvular apparatus makes TMVRpl much more challenging than its counterpart on aortic valve position.

A meticulous angio-CT study is mandatory in the screening phase and should evaluate:

(1) the functional anatomy of the left ventricular outflow tract (LVOT) with the presence of a mitral aortic angle $>120^{\circ}$ and the absence of severe septal hypertrophy to avoid a neo-LVOT obstruction, the most dreaded complication; 
(2) an adequate annular size, which must not be too small not to allow safe anchoring and not too wide to increase the risk of LVOT obstruction;

(3) the LV function, which could be greatly reduced after TMVRpl;

(4) the native leaflets and the eventual annular calcifications to find the best implant site, which should be as atrial as possible.

As the transfemoral approach is under development, the patients to be screened must be able to tolerate transapical access. This condition may constitute a great limitation, especially in patients with SMR who have remodeled LVs or ischemic scars.

Structural valve deterioration is a great concern for TMVRpl. The systolic pressure gradient generates a significant mechanical stress on the bioprosthetic mitral valve, resulting in a more frequent degeneration if compared to the aortic counterpart. This rate is increased even more by the young age of the patients $(\sim 10$ years younger than recipients of aortic valves $)^{[17]}$. Indeed, greater hemodynamic shear stress, differences in calcium deposition, and residual leaflet antigenicity contribute to a higher tendency of valve degeneration in younger patients ${ }^{[18]}$.

Another factor to be considered is the feasibility of anticoagulation treatment to avoid device thrombosis. This complication was reported in $3 \%$ of patients in TMVRpl studies ${ }^{[19]}$, which adopted a minimum regimen of three months of anticoagulation mimicking the recommendations from current guidelines regarding conventional surgical mitral valve replacement.

A careful analysis of the enrollment criteria of the TMVRpl trials allows us to identify the patient to be denied or offered this therapy [Table 1]. The presence of a LV (LV end diastolic diameter $>70 \mathrm{~mm}$, LVEF $<30 \%$ ) or right ventricle (RV) dysfunction, hypertrophic obstructive cardiomyopathy, and restrictive or constrictive cardiomyopathy represent common exclusion criteria [Figure 3]. Comorbidities such as severe chronic kidney disease requiring dialysis, significant coronary artery disease, and bleeding or thrombotic disorders are further unfavorable factors. Regarding the valve apparatus, predicted LVOT obstruction, severe mitral stenosis, fused commissures, prohibitive mitral annulus calcifications, and vegetations or valvular masses have to be avoided. The most important features that favor TMVRpl over TMVR are represented by the prediction of a suboptimal MR reduction with the latter technique and some of the TMVR exclusion criteria such as multisegment or commissural disease, perforations, or clefts. For the reasons outlined above, the ideal patient should have a good LV function (LVEF > 30\%) in the absence of scars or remodeled LV walls that could be irreversibly damaged by a transapical access.

\section{FUTURE PERSPECTIVES}

Currently, a repair-first strategy is the transcatheter way to go for patients with severe MR. The lessons learned from the surgical experience and the paucity of data on TMVRpl make this approach a second choice $^{[20]}$. A percutaneous repair respects the complex anatomy and physiology of the mitral valve. The preservation of the valvular and subvalvular apparatus leads to LV and RV reverse remodeling with improved functional performance ${ }^{[21,22]}$. However, the technological progress in transcatheter valves could increase the appeal of TMVRpl therapy if a valve capable of adapting to such variable anatomies was found. This hoped-for result would mean having a device less technically demanding, with great versatility and more predictable results in terms of MR reduction.

Transseptal delivery systems for TMVRpl are emerging and will replace the current transapical approach, which is burdened by an increased risk of peri-procedural complications. 


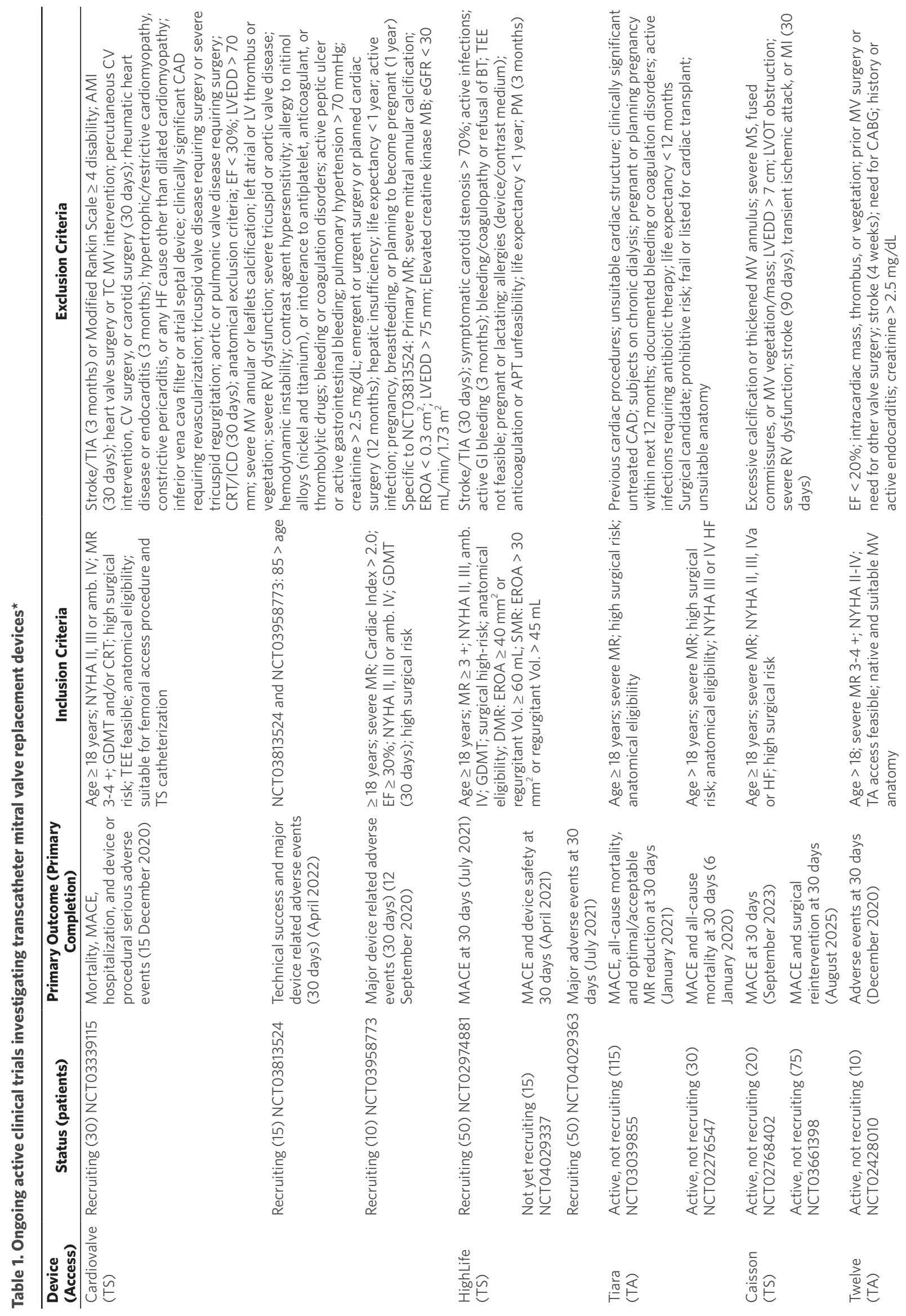




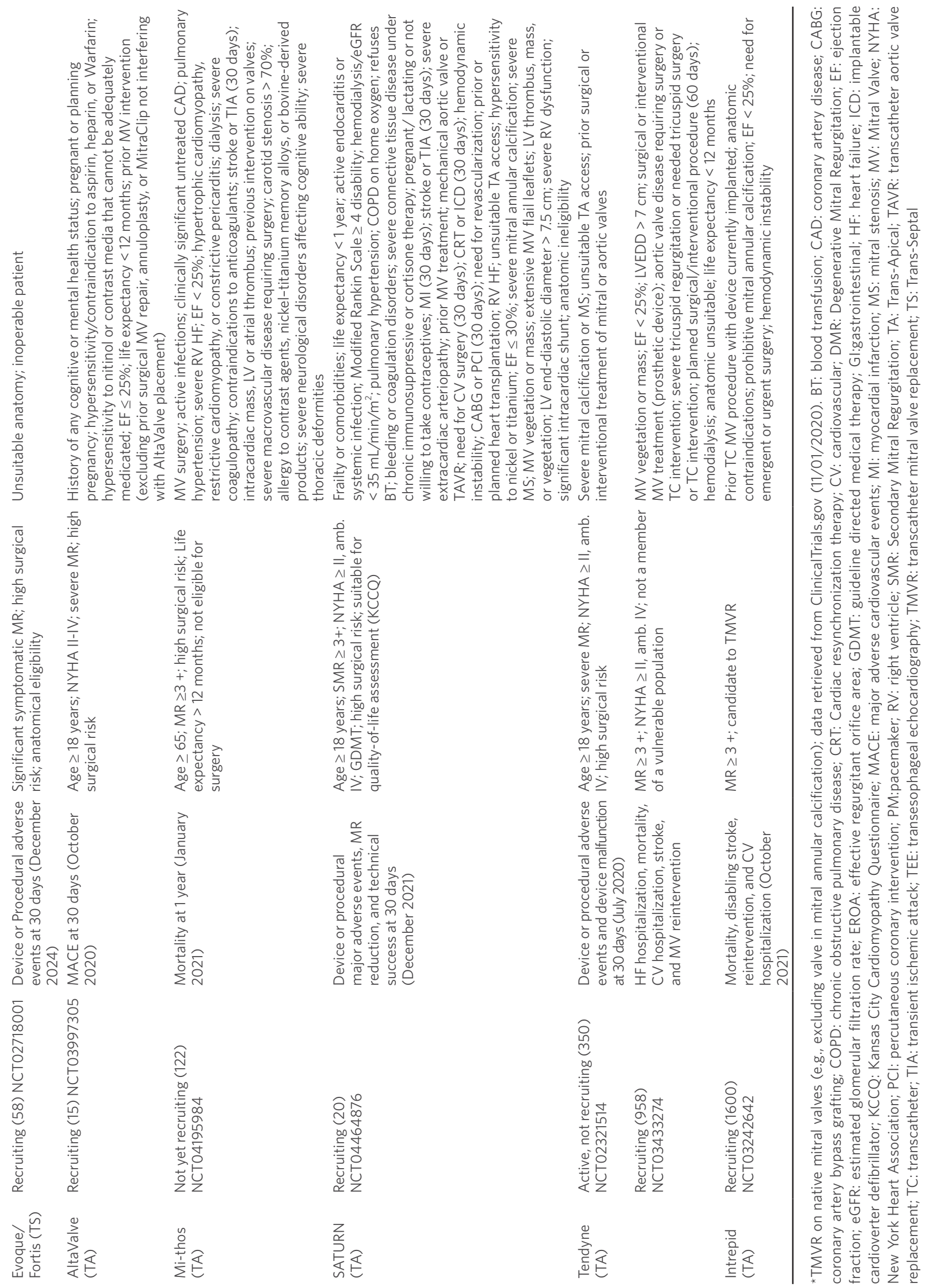


Similar to aortic bioprosthetic valves, TMVRpl devices need an assessment of the rates of structural degeneration, prosthesis-related endocarditis, and thromboembolic/hemorrhagic events. Studies on longterm clinical outcomes and trials on optimal antithrombotic therapy will provide these data.

While evidence on the use of TMVR for advanced HF is being published ${ }^{[13,14]}$, whether TMVRpl may play a role in this setting has yet to be proved. Complete and more reproducible reductions in MR may allow for an uptitration of heart failure medical therapies, and this gain could lead to even greater clinical benefits.

Studies on TMVRpl available thus far are promising in terms of feasibility and efficacy, but they report high rates of peri-procedural complications and all-cause mortality (technical success $91.7 \%$, stroke $2.9 \%$, unplanned mitral valve surgery $4 \%$, and 30 -day mortality $13.6 \%)^{[19]}$. For this reason, the clinical applicability of these procedures is limited and finds room when TMVR is contraindicated. These data are influenced by two critical factors:

(1) The patients enrolled in TMVRpl studies are elderly, have a relevant comorbidity burden and very high operative risks, or are considered inoperable (compassionate use ${ }^{[19]}$.

(2) A quite selective process limits the anatomical eligibility to TMVRpl and consequently the enrollment rate; screening failure is reported as up to $70 \%{ }^{[23,24]}$.

The great potential of these procedures and their theoretical applicability will be proved in the coming years when more scientific data become available.

\section{CONCLUSION}

Technical and more so technological advances have forged a vast armamentarium of transcatheter mitral valve therapies. While some TMVR devices (e.g., MitraClip) have large supporting studies, TMVRpl devices need more evidence. Clinical investigations, in the form of randomized trials, will define the specific role and the ideal target population of TMVRpl. At the moment, for patients with severe MR, a repair-first strategy is the preferable choice, and a replacement technique finds applicability in highly selected cases. As soon as TMVRpl obtains the necessary confirmations to be considered a complementary strategy to TMVR, a substantial number of transcatheter options will be available to handle mitral valve disease. A one-device-for-all paradigm is not realistic; having a complete mitral toolbox will be fundamental to address all the different pathologies, mechanisms, and patients to allow specific patienttailored approaches.

\section{DECLARATIONS}

\section{Authors' contributions}

Conceived and designed the study, performed data interpretation, and were involved in all the phases of the writing process: Scotti A, Galasso M, Margonato A, Godino C

\section{Availability of data and materials}

Not applicable.

\section{Financial support and sponsorship}

None.

\section{Conflicts of interest}

All authors declared that there are no conflicts of interest. 


\section{Ethical approval and consent to participate}

Not applicable.

\section{Consent for publication}

Not applicable.

\section{Copyright}

(c) The Author(s) 2021.

\section{REFERENCES}

1. Feldman T., Wasserman HS., Herrmann HC., et al. Percutaneous mitral valve repair using the edge-to-edge technique: six-month results of the EVEREST Phase I Clinical Trial. J Am Coll Cardiol 2005;46:2134-40.

2. Puls M., Lubos E., Boekstegers P., et al. One-year outcomes and predictors of mortality after MitraClip therapy in contemporary clinical practice: Results from the German transcatheter mitral valve interventions registry. Eur Heart J 2016;37:703-12.

3. Maisano F., Franzen O., Baldus S., et al. Percutaneous mitral valve interventions in the real world: early and 1-year results from the ACCESS-EU, a prospective, multicenter, nonrandomized post-approval study of the Mitraclip therapy in Europe. J Am Coll Cardiol 2013;62(12):1052-61.

4. Praz F., Spargias K., Chrissoheris M., et al. Compassionate use of the PASCAL transcatheter mitral valve repair system for patients with severe mitral regurgitation: a multicentre, prospective, observational, first-in-man study. Lancet 2017;390:773-80.

5. Glower DD., Kar S., Trento A., et al. Percutaneous mitral valve repair for mitral regurgitation in high-risk patients: Results of the EVEREST II study. J Am Coll Cardiol 2014;64:172-81.

6. Lim DS., Reynolds MR., Feldman T., et al. Improved functional status and quality of life in prohibitive surgical risk patients with degenerative mitral regurgitation after transcatheter mitral valve repair. J Am Coll Cardiol 2014;64:182-92.

7. Deferm S., Bertrand PB., Verbrugge FH., et al. Atrial functional mitral regurgitation: JACC review topic of the week. J Am Coll Cardiol 2019;73:2465-76.

8. Packer M., Grayburn PA. New evidence supporting a novel conceptual framework for distinguishing proportionate and disproportionate functional mitral regurgitation. JAMA Cardiol 2020: 469-75.

9. Baumgartner H., Falk V., Bax JJ., et al. 2017 ESC/EACTS Guidelines for the management of valvular heart disease. Eur Heart $J$ 2017;38:2739-86.

10. Stone GW., Lindenfeld JA., Abraham WT., et al. Transcatheter mitral-valve repair in patients with heart failure. $N$ Engl $J$ Med 2018;379:2307-18.

11. Obadia JF., Messika-Zeitoun D., Leurent G., et al. Percutaneous repair or medical treatment for secondary mitral regurgitation. $N$ Engl $J$ Med 2018;379:2297-306.

12. Bonow RO., O'Gara PT., Adams DH., et al. 2020 focused update of the 2017 ACC expert consensus decision pathway on the management of mitral regurgitation: a report of the American College of Cardiology Solution Set Oversight Committee. J Am Coll Cardiol 2020;75:2236-70.

13. Scotti A., Margonato A., Godino C. Percutaneous mitral valve repair in patients with secondary mitral regurgitation and advanced heart failure. Mini-Invasive Surg 2020;4:49.

14. Godino C., Munafò A., Scotti A., et al. MitraClip in secondary mitral regurgitation as a bridge to heart transplantation: 1-year outcomes from the International MitraBridge Registry. J Hear Lung Transplant 2020;39:1353-62.

15. Feldman T., Cilingiroglu M. Percutaneous leaflet repair and annuloplasty for mitral regurgitation. J Am Coll Cardiol 2011:529-37.

16. Lubos E., Schlüter M., Vettorazzi E., et al. MitraClip therapy in surgical high-risk patients: Identification of echocardiographic variables affecting acute procedural outcome. JACC Cardiovasc Interv 2014;7:394-402.

17. Brown JM., O’Brien SM., Wu C., Sikora JAH., Griffith BP., Gammie JS. Isolated aortic valve replacement in North America comprising 108,687 patients in 10 years: changes in risks, valve types, and outcomes in the Society of Thoracic Surgeons National Database. $J$ Thorac Cardiovasc Surg 2009;137:82-90.

18. Mylotte D., Andalib A., Thériault-Lauzier P., et al. Transcatheter heart valve failure: a systematic review. Eur Heart J 2015;36:1306-27.

19. Del Val D., Ferreira-Neto AN., Wintzer-Wehekind J., et al. Early experience with transcatheter mitral valve replacement: a systematic review. J Am Heart Assoc 2019;8:e013332.

20. Taramasso M., Gavazzoni M., Nickenig G., Maisano F. Transcatheter mitral repair and replacement: which procedure for which patient? EuroIntervention 2019;15:867-74.

21. Adamo M., Godino C., Giannini C., et al. Left ventricular reverse remodelling predicts long-term outcomes in patients with functional mitral regurgitation undergoing MitraClip therapy: results from a multicentre registry. Eur J Heart Fail 2018;21:ejhf.1343.

22. Godino C., Salerno A., Cera M., et al. Impact and evolution of right ventricular dysfunction after successful MitraClip implantation in patients with functional mitral regurgitation. IJC Hear Vasc 2016;11:90-8.

23. Bapat V., Rajagopal V., Meduri C., et al. Early experience with new transcatheter mitral valve replacement. $J$ Am Coll Cardiol 2018;71:12-21.

24. Sorajja P., Moat N., Badhwar V., et al. Initial feasibility study of a new transcatheter mitral prosthesis: the first 100 patients. $J$ Am Coll Cardiol 2019;73(11):1250-60. 